\title{
Evaporation of Sessile Droplets on Nano-porous Alumina Surfaces
}

\author{
Sanchit K. Singh ${ }^{1}$, Dheeraj Pratap ${ }^{2}$, S. Anantha Ramakrishna ${ }^{3}$, Sameer Khandekar ${ }^{4}$ \\ 1: Department of Mechanical Engineering, Indian Institute of Technology Kanpur, India, sanchits@iitk.ac.in \\ 2: Department of Physics, Indian Institute of Technology Kanpur, India, pdheeraj@iitk.ac.in \\ 3: Department of Physics, Indian Institute of Technology Kanpur, India, sar@iitk.ac.in \\ 4: Department of Mechanical Engineering, Indian Institute of Technology Kanpur, India, samkhan@iitk.ac.in
}

\begin{abstract}
An experimental investigation of evaporation of sessile droplets is presented on nano-porous alumina surfaces with different pore distribution morphologies and pore sizes. Evaporation can be considered as a quasi-steady-state process, such that the vapor concentration distribution above the droplet satisfies the Laplace equation, but with a time-varying droplet surface. For benchmarking, the evaporation of sessile water and ethanol droplets is also investigated on standard borosilicate glass and Teflon surfaces respectively, and results are compared with the previous work of Picknett and Bexon (1977). Contact angle variation with time is also recorded and high speed videos showing the spreading process of droplets on nano-porous surfaces are taken. The results clearly show that nano-structuring is an effective tool to control wettability as well as the diffusive evaporation process.
\end{abstract}

Keywords: Droplet evaporation, Diffusion, Nanostructured surfaces, Spreading, Wettability

\section{Introduction}

In recent years, due to the advent of nanotechnology, the deceptively simple problem of droplet evaporation is again attracting attention (Erbil, 2012). From a fundamental, as well as practical viewpoint, three configurations of droplet evaporation are of interest: (i) evaporation of simple fluids on plain surfaces; (ii) evaporation of nanofluids (simple fluids containing suspended nanoparticles on plain surfaces and; (iii) evaporation of simple fluids on prefabricated micro/nanostructured surfaces. While the first case represents the classical problem of droplet evaporation, wherein decent amount of literature already exists, the latter two cases are not yet comprehensively explored. In this paper we focus our attention on case (iii).

A theoretical study of first case, i.e., of simple fluids evaporating on plain surfaces, has been done analytically by Picknett et al. (1977) by using an analogy between electrostatic potential of top half of an equiconvex lens and the diffusive droplet evaporation. Two modes of evaporation were distinguished: (i) at constant contact angle, with diminishing base contact area and; (ii) at constant base contact area with diminishing contact angle. $\mathrm{Hu}$ and
Larson (2002) numerically solved the vapor concentration distribution and the evaporation flux using finite element method. The evaporation flux calculated in the numerical analysis was fitted by the form of equation suggested by Deegan et al. (1997). Approximate expressions for the evaporation flux and evaporation rate were derived.

The case (ii) of evaporating drops containing colloidal particles on a solid substrate can be used for dispensing or organizing small particles suspended within them (e.g. see Bhardwaj, 2010). The dried deposit is not always uniform. This resulting structure is referred as a coffee-ring pattern, and is caused by enhanced evaporation at the wetting line, as explained by Deegan et al. (1997). Interactions between agglomerating particles at the wetting line and the wetting line receding during evaporation can also form patterns involving multiple rings (Shmuylovich et al., 2002).

In biological applications, liquid droplet evaporation can be used to comb DNA, as proposed by Bensimon et al. (1995). A DNA strand attached by one end to a glass surface stretches progressively as the liquid interface retreats during evaporation. When all the liquid vanishes, the DNA strand forms a linear 
segment on the glass plate. In the field of microelectronics, evaporating drops containing colloidal particles can be used to manufacture nanowires. For example, printing of fine copper lines by a solution of copper hexanoate in isopropanol or choloroform (Ondarcuhu and Joachim, 1998). Evaporating drops containing colloidal particles have been used to deposit zeolite nano crystals on silicon wafers to form nanoporous films with characteristic features size of $5-10 \mu \mathrm{m}$, to deposit variety of nanoparticles (e.g. cadmium sulphide, silver, cobalt and ferrite nanocrystals and gold nanoparicles) from organic solvents (Wang et al., 2001). Varying the particle size changes the wavelength of light emitted by particles in a wide spectral range. This can be utilized in a variety of light emitting and processing devices. Photo-lithography is another area of application in microelectronics where latex particles are deposited on a silicon substrate and can be used as an etching mask, which is a component used for fabricating microelectronics devices (Burmeister et al., 1999).

Evaporation of droplets of simple fluid on surfaces with nano-projection (case (iii)) was studied recently by Choi and Kim (2009) and on Cu-based hydrophobic surfaces by Lee, Zhang and Kim (2012). The work done so far focuses on the evaporation dynamics on surfaces with nano-projection, however, evaporation on nanoporous surfaces is yet to be fully explored. In this paper, we focus our attention on evaporation of simple fluids on prefabricated micro/nano-structured surfaces. The purpose of the present work is to study how nano-porous surfaces alter the wettability, spreading, equilibrium contact angle and rate of evaporation of sessile droplets of simple fluids, subjected to controlled thermo-mechanical boundary conditions.

\section{Theory}

The diffusion equation for evaporation of a droplet is given by:

$$
\frac{\partial c}{\partial t}=D \cdot \nabla^{2} c
$$

where, $c$ is the local vapor mass concentration and $D$ is the binary diffusion coefficient of the evaporating liquid in the medium. The time required for the vapor concentration to adjust to changes of the droplet shape and surface temperature is on the order of $\left(r_{b}\right)^{2} / D_{A B}$ (Hu et al. 2002), which is three orders of magnitude smaller than the total evaporation time of the micro-liter sized drops considered in this study. Therefore, the vapor concentration evolves in a quasi-steady manner with respect to the drop evaporation and we neglect the term on the left hand side of the above equation. The boundary conditions for eq. 1 are:

$\partial c / \partial z=0$ at $r>r_{b}, z=0$;

(no penetration of vapor in the substrate)

$\partial c / \partial z=0$ at $z>h_{\max }, r=0$;

$c=c_{\infty}$ at $r=\infty, z=\infty$;

$c=c_{\text {sat }}$ at the interface.

The general diffusion rate equation for a sphere kept in an infinite medium, relating the concentration of vapor with the radial position, by assuming that the vapor concentration at the wet surface of the sphere, $c_{s}$ is equal to its equilibrium concentration, is given by Maxwell (1890):

$\frac{d m}{d t}=4 \pi \cdot R_{s} \cdot D\left(c_{s}-c_{\infty}\right)$

where, $c_{\infty}$ is the concentration of the vapor at infinite distance from the drop then, we have the following boundary conditions, $c=c_{s}$ when $r=R_{s}$ and $c=c_{\infty}$ when $r=\infty$. An analogy between electric potential and diffusive flux can be invoked to calculate the rate of evaporation in case of a body of any shape:

$\frac{d m}{d t}=4 \pi \cdot D \cdot C_{x}\left(c_{s}-c_{\infty}\right)$

where, $C_{x}$ is electrostatic capacitance of an isolated body.

The evaporation of a sessile drop can be evaluated by calculating the capacitance of an isolated conducting body of the same size and shape as the drop, i.e. determining the capacitance of the equiconvex lens formed by the sessile drop and its image in the substrate surface (Fig. 1). The evaporation rate of this lens must, by symmetry, be twice that of the sessile drop, and so the lens capacitance must be twice that of the body equivalent to the drop: 


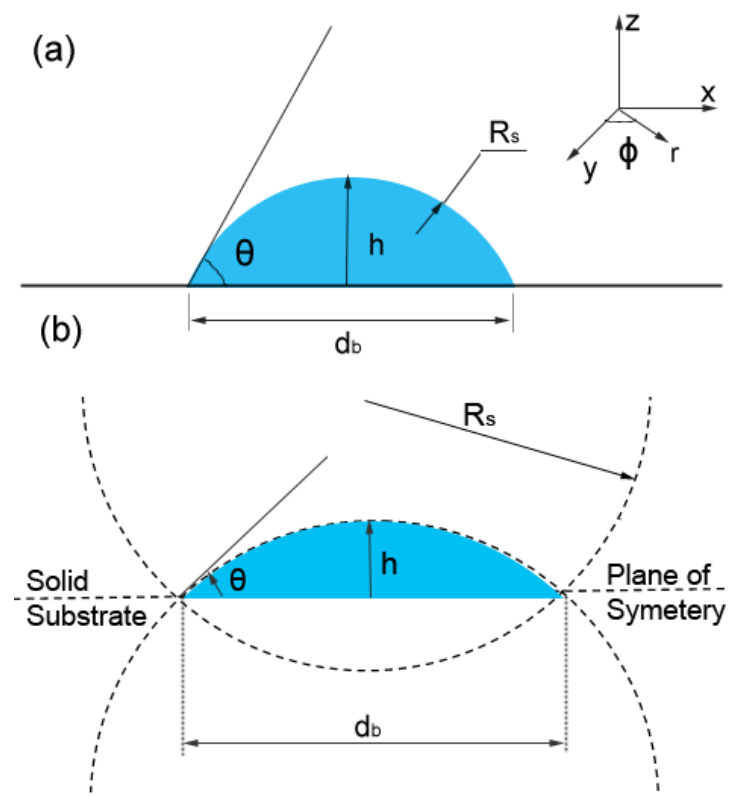

Fig. 1: (a) Profile of a droplet on a surface depicting the details of the coordinate system (b) Shows two intersecting spheres forming an equi-convex lens. A droplet on a solid surface can be taken as half of this lens across the axis of symmetry, as shown.

$C_{\text {sessile drop }}=C / 2$

where, $C$ is the capacitance of the lens.

The series solution proposed by Snow (1949) that relates apex angle of the lens to relate the capacitance can be approximated to be:

For $0 \leq \theta \leq 0.175$ radians:

$C / r=0.6366 \theta+0.09591 \theta^{2}-0.6144 \theta^{3}$

and, for $0.175 \leq \theta \leq \pi$ radians:
$C / r=0.00008957+0.6333 \theta+0.1160 \theta^{2}$

$0.088783 \theta^{3}+0.01033 \theta^{4}$

The rate of evaporation of a sessile droplet with pinned contact line can be evaluated by (Picknett et al. (1977)):

$$
\frac{d W}{d t}=-\frac{k E W^{\circ / 3}(C / r) \sin \theta^{\circ}}{2 \rho^{2 / 3} \sin \theta}
$$

where, $W^{\circ}$ is the initial mass of the droplet, $k=4 \pi D\left(c-c_{s}\right)$ and $E^{3}=3 /\left\{\pi(1-\cos \theta)^{2}(2+\cos \theta)\right\}$.

\section{Experiment}

\subsection{Details of Set-up and Sample Surfaces}

The schematic of the experimental setup is shown in fig. 2(a). Atmospheric air is pressurized using a compressor. It is then passed through a dehumidifying chamber which contains liquid Nitrogen. The dry air thus obtained is passed through a temperature controller. The conditioned dry air is then divided into two parts, one of which is bubbled through a water bath, which ensures complete saturation of the air. Finally, the two isothermal air streams i.e., fully dry and fully saturated, are mixed in suitable proportions to get air at the desired RH to the test cell.

The control volume consists of a cubical chamber $(8 \mathrm{~cm} \times 4 \mathrm{~cm} \times 4 \mathrm{~cm})$, the size of which is sufficiently large as compared to the size of the droplet, hence, satisfying the (a)

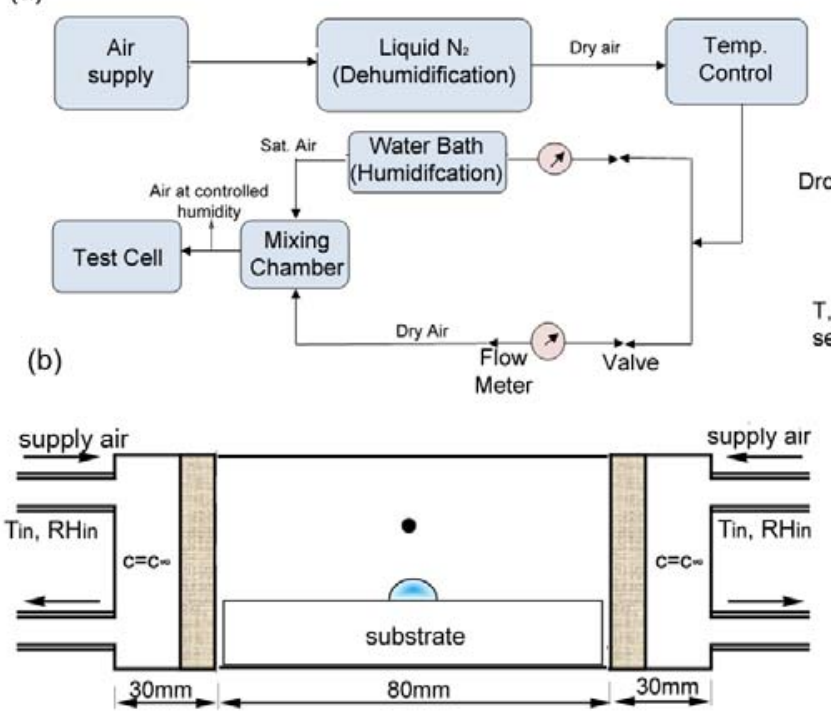

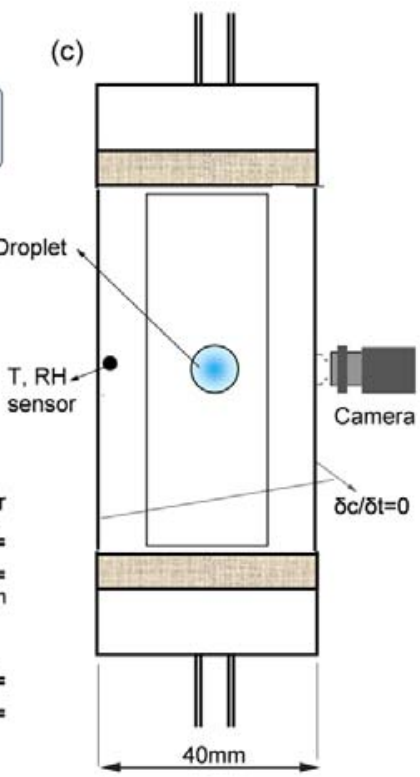

Fig. 2: (a) Shows the basic sketch of the experimental set-up, (b) Side view of the test cell with dimensions, (c) Top view of the test cell with position of camera. 
conditions at $r=\infty$. Two sides of the control volume are exposed to constant humidity levels throughout the experiment, via a thick porous layer of paper; the remaining four sides have insulted boundary conditions. Arrangement is such that there is no bulk convection inside the test cell; evaporative transport is diffusive. The chamber is fitted with a combined temperature and humidity sensor (GE-ChipCap ${ }^{\mathbb{B}}$ ). An isolated liquid droplet is placed at the centre of the control volume using a micro-syringe and temporal variation of contact angle is captured by digital videography.

For all experiments, deionized, degassed and filtered water is used. Glass and Teflon substrates are cleansed with bleaching powder and then treated with $5 \%$ ethanol solution in ultrasonic bath for $10 \mathrm{~min}$. to remove any kind of surface impurities. Nano-porous alumina surfaces are prepared (see next section) with different pore sizes and morphologies.

The high speed camera used for videography the has the capability to capture videos at pixel resolution of $1024 \times 1024$ at frame rates up to 2,000 fps (Fastcam-Photron ${ }^{\circledR}$ SA-3).

\subsection{Preparation of Anodized Nanoporous Alumina (ANA) surfaces}

The following three morphologies of pores have been manufactured and used:

- Randomly ordered nanopores

- Linearly aligned nanopores

- Organized hexagonal nanopores

Anodized Nanoporous Alumina (ANA) was prepared using pure aluminum foil $(99.9 \%$ Loba Chemie $^{\circledR}$ and $99.999 \%$ Sigma-Aldrich $^{\circledR}$ ). The aluminum foils of about $100-125 \mu \mathrm{m}$ thickness are first cleaned with acetone. The pure aluminum results in pores forming on a highly organized hexagonal lattice while the less pure one results in a more random formation of the pore. The rolled aluminum foils have micro-and nano-scratches as well and are anisotropically stressed along the rolling direction. This feature could be used to linearly organize the nanopores in that direction. Anodization of the cleaned aluminum with $99.9 \%$ purity was carried out in a solution of oxalic acid ( 0.3 molar) at $40 \mathrm{~V}$ for 12 hours at $18^{\circ} \mathrm{C}$ for obtaining the ANA with linearly organized pores.
For forming the pores in other morphologies, the cleaned aluminum foils of both purity levels are first electro-polished in an electrolyte made of a mixture of ethanol and perchloric acid (5:1 volume) at a current density of $0.076 \mathrm{~A} / \mathrm{cm}^{2}$ for around $5 \mathrm{~min}$. and surfaces with mirror like finish is obtained. This resulted in the removal of most of the surface scratches and stresses. For obtaining the ANA with pores organized in a random manner, a first anodisation of the electropolished aluminum foil of $99.9 \%$ purity in oxalic acid ( 0.3 molar) at $40 \mathrm{~V}$ and $18^{\circ} \mathrm{C}$ for 12 hours is sufficient. For the ANA with hexagonally organized pores, the electropolished aluminum foil of purity $99.999 \%$ is first anodized in solution of oxalic acid $(0.3$ molar) at $40 \mathrm{~V}$ for 3 hours at $0^{\circ} \mathrm{C}$. The formed alumina is etched off in a mixture of phosphoric acid, chromic acid and water (3.5 $\mathrm{ml} \mathrm{H}_{3} \mathrm{PO}_{4}, 4.5 \mathrm{gm} \mathrm{CrO}_{3} / 200 \mathrm{ml}$ DI water solution) at $90^{\circ} \mathrm{C}$ for around $45 \mathrm{~min}$. A second anodization for 12 hours keeping the rest of the parameters same as before resulted in highly organized nanopores in a hexagonal lattice.

While the periodicity (or average pore spacing) depends primarily on the nature of the acid and $\mathrm{pH}$ during anodization, the pore size can be changed after anodization by further etching. We used samples with two kinds of pore sizes for this study - small ones with an average pore size of $(70 \mathrm{~nm})$ and larger pores of average pore size $(120 \mathrm{~nm})$ by pore widening in a solution of 5\% phosphoric acid for $90 \mathrm{~min}$. The average pore spacing in our samples is $120 \mathrm{~nm}$.

\subsection{Procedure and Data Reduction}

Firstly, the spreading and subsequent attainment of equilibrium of small micro-sized droplets is recorded by high speed videography. This reflects the inherent spreading and wetting characteristics of the particular substrate under consideration.

Subsequently, evaporation of a $5 \mu$ sessile droplet of water on plain glass and Teflon surface is undertaken. For both cases the droplet remains pinned to the surface throughout the evaporation time. The apparent contact angle of the droplet is monitored throughout the course of evaporation at 
different humidity levels. Also, the rate of evaporation of a sessile droplet of ethanol on glass is studied for benchmarking with the existing models. In the next part, evaporation of water droplet is investigated on the nanoporous alumina surfaces having different pore distribution morphologies and pore sizes. For the purpose of measuring the equilibrium contact angle a simple, yet powerful tool in ${\text { Image }{ }^{\circledR}}^{\circledR}$ software, known as DropSnake, which is based on B-spline snakes (active contours) to shape the drop, is used.

A code is developed based on the theory given by Picknett et al. (1977) (eq. 5) to calculate the time taken by the droplet to evaporate completely, subjected to the experimental conditions. The results thus obtained are compared.

\section{Results and Discussions}

Fig. 3a-d shows the spreading dynamics of a $5 \mu \mathrm{l}$ water droplet on a glass substrate, plain alumina surface, alumina surface with randomly distributed nanopores and, organized hexagonal nanopores, respectively. The final equilibrium angle obtained is $44.6^{\circ}$ for glass, $51.2^{\circ}$ for plain alumina and, as can be seen from the figure, $12.1^{\circ}$ for the substrate with organized nanopores. The droplet completely spreads on surface with randomly distributed pores. Thus, the nanoporous structure drastically affects the wettability of the substrate.

(a)

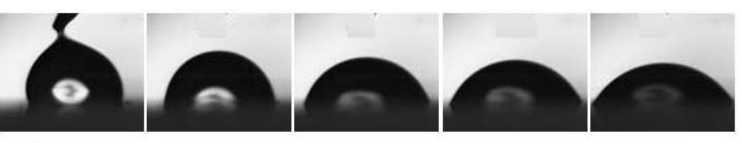

(b)

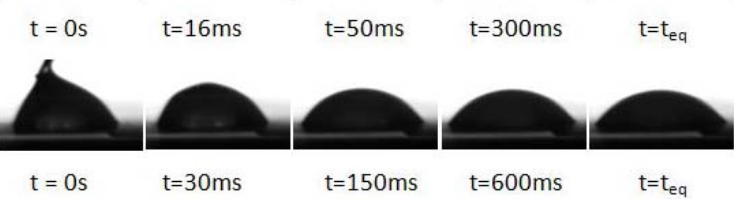

(c)

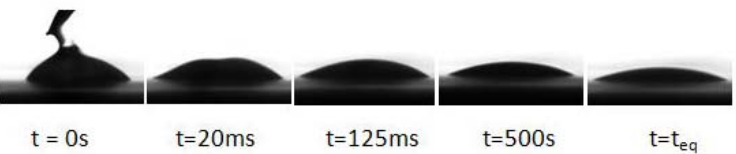

(d)

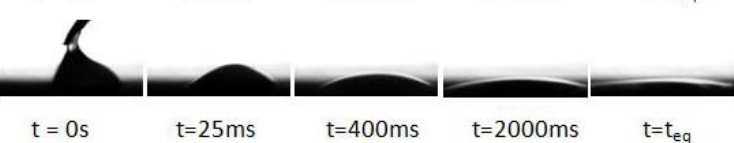

Fig. 3: Spreading dynamics of a $5 \mu 1$ droplet on (a) a glass surface; (b) a plain alumina surface; (c) an alumina surface with organized nanopores and; (d) an alumina surface with randomly distributed nanopores, at different time intervals at at 2000 fps using a high speed camera (magnification factor is different for different images to accommodate the final sizes).
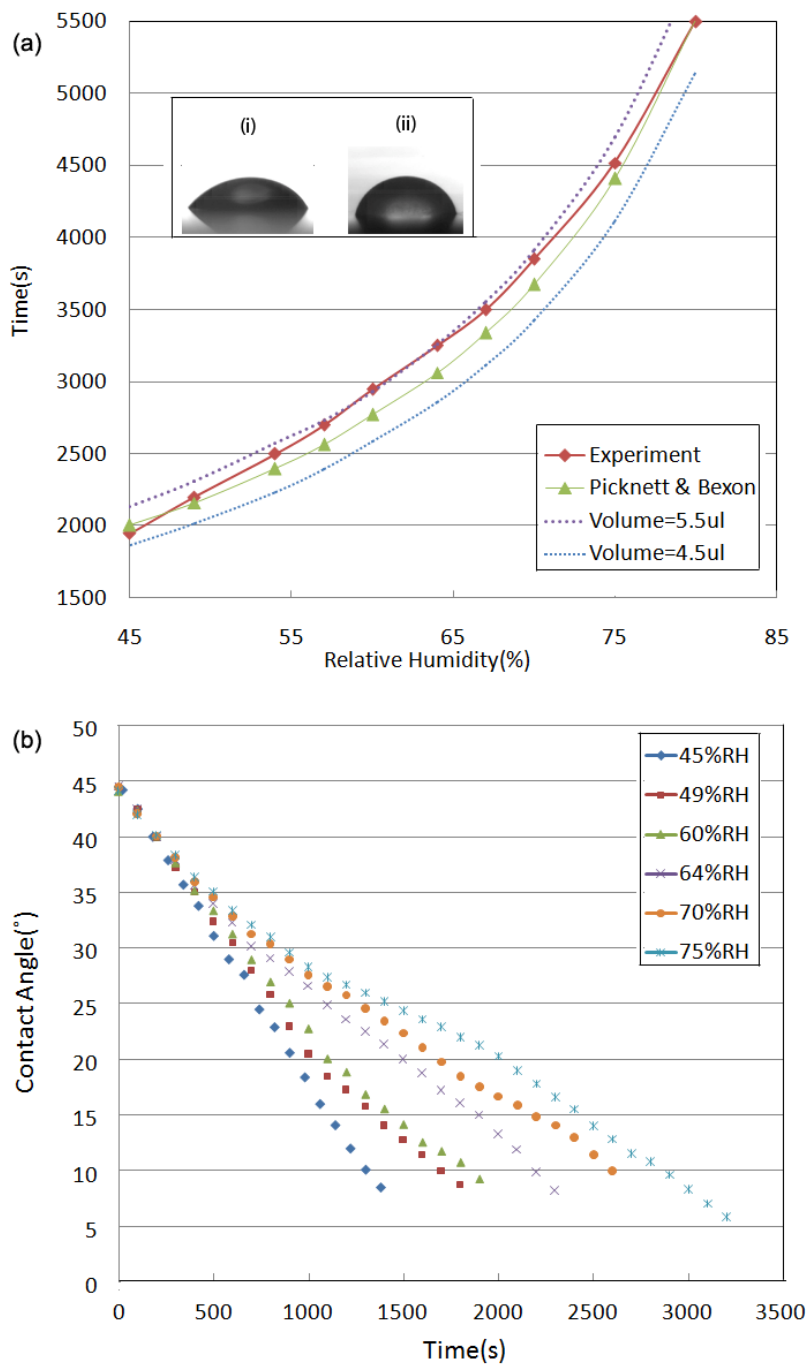

Fig. 4: (a) Time taken by $5 \mu 1$ water droplet to evaporate on a glass surface at different relative humidity. The results from theoretical model is also present for $4.5,5,5.5 \mu$ d droplets. In inset (i) $5 \mu \mathrm{l}$ droplet of water on glass with a contact angle of $44.6^{\circ}$, (ii) $5 \mu \mathrm{l}$ droplet of water on Teflon with a contact angle of $73.2^{\circ}$; (b) Shows contact angle variation of a evaporating 5 $\mu \mathrm{l}$ water droplet after different humidity levels.

Fig. 4(a) presents the result obtained from analytical model given by Picknett et al. (1977) and the results obtained from experiments on glass and Teflon. It can be observed that the results agree quite well with the model with minor discrepancy. This can be attributed to the fact that while the theory is for diffusion in infinite medium, the experimental test cell is of finite size. The model works well for other simple fluids like ethanol evaporating on glass as well. Experimentally it is observed that an ethanol droplet of $5 \mu \mathrm{l}$ with an initial contact angle of $21.2^{\circ}$ takes about 420 s to completely evaporate. The results obtained from the models gives an evaporation time of about $395.2 \mathrm{~s}$ which comes to be within an error band of about $5.7 \%$. 
The observed initial contact angle of a water droplet on glass $\left(\sim 44.6^{\circ}\right)$ is irrespective of the change in RH of the vicinity as seen in fig. 4(b). The variation of contact angle with time at various humidity levels is also shown in the figure. It is observed that the variation of contact angle is nonlinear and the nonlinearity increases as the RH increases. Another interesting phenomenon to be observed is that the contact line remains pinned for major part of the time and de-pinning occurs only after the contact angle becomes less than about $8^{\circ}$.

Fig. 5(a) shows the variation of humidity and temperature as the water droplet evaporated on a nanoporous surface at $45 \%$ base RH. It is observed that the rise in humidity level is detected by the sensor soon as the droplet is placed on the surface. The RH keeps rising for the initial few minutes; becomes stable for some time and then, starts gradually falling back to base level towards the end. The recorded $\mathrm{RH}$ inside the chamber takes about 1 min. to fall back to base level after the drop has visually disappeared. However, no significant change in temperature is observed.

The model by Picknett et al. (1977) fails on the nanoporous surface as the droplet spreads nearly completely on such surfaces. It is observed that the spreading and wettability increase as the orderliness of pore distribution decreases, which eventually leads to a high rate of evaporation (Fig. 5(b)). The droplet spreads as soon as it is kept on the surface with random distribution of pores, losing its identity completely.

However, it retains its shape with a very low contact angle of about $12^{\circ}$ on a surface with ordered pores. Hence, the rate of evaporation tends to be highest on the surface with randomly oriented pores followed by surface with linearly aligned pores and surface with organized hexagonally pores. Another interesting observation is that, irrespective of the distribution of pores, the rate of evaporation is correspondingly higher when pore size is larger.

Complications arise in the problem of evaporation because the shape of the droplet during the evaporation is a priori unknown, and also because of a number of special effects that have to be taken into account. For instance,
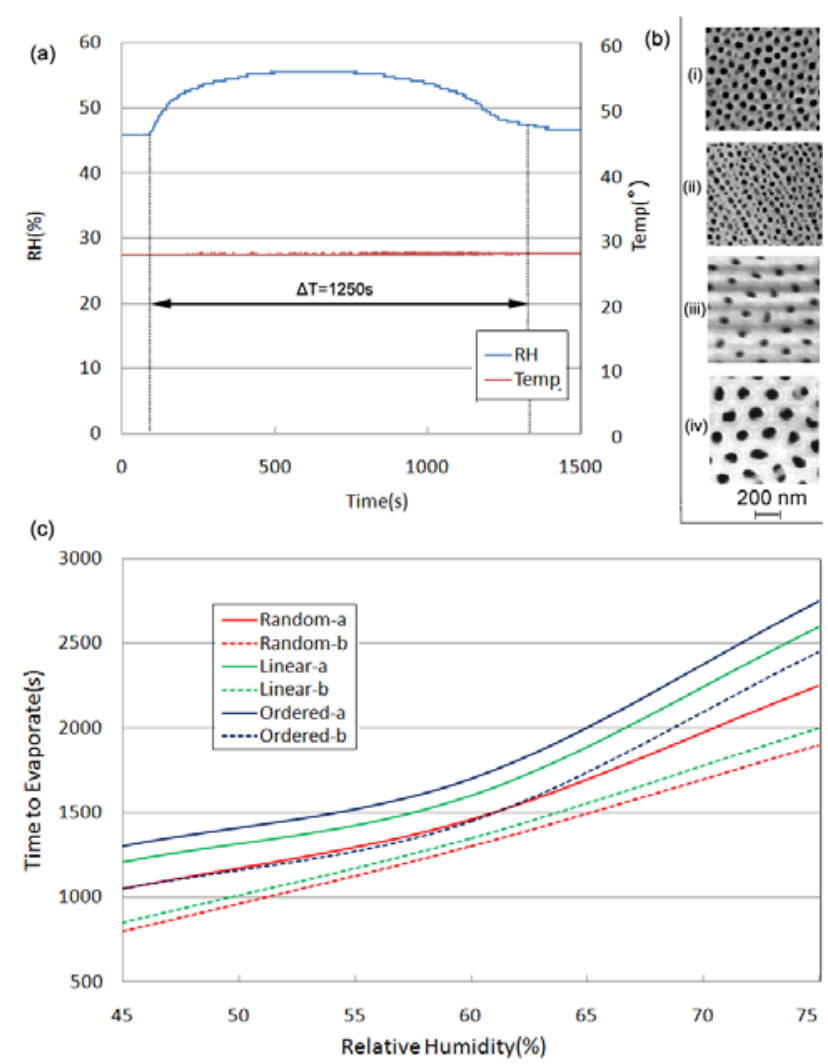

Fig. 5: (a) Shows the variation of temperature and relative humidity inside the control volume when a droplet evaporates on a nano-pored surface; (b)-(i),(ii),(iii) SEM images of surfaces with randomly, linearly ordered and organized nanopores and, (iv) Surface with ordered pores, after pore widening; (c) Time taken to evaporate a $5 \mu$ water droplet on the three nanoporous alumina surfaces. The dotted lines for each case show the results from larger pore size.

evaporation of the droplet generally leads to a decrease of the temperature inside it. Due to this, a heat flux can be generated from the substrate into the droplet. Other effects, like the Marangoni effect, also come into play and complicate the situation further. These effects need further exploration.

\section{Conclusions}

It can be inferred from the results as well as the theory that the rate of evaporation of a droplet on a surface does not decrease linearly with increase in relative humidity. The rate of evaporation depends on the initial contact angle and radius of the droplet. Hence, the rate of evaporation of a Teflon surfaces is much lesser as compared to the rate of evaporation on glass.

Also, the change of contact angle with time shows a non-linear variation at all RH levels.

Pore size and its distribution morphologies significantly affect the evaporation dynamics of a droplet. The contact angle and spreading on such surfaces is much more as compared to 
plain surfaces of glass and Teflon. These effects are more pronounced when there is more randomness in the distribution of these pores. Also, increasing the nano-pore size has a similar effect.

Future work on this may include the coupling of energy equation with diffusive process as there is a significant drop in temperature of the drop as it evaporates. Also, the study can be extended to evaporation of colloidal fluids with nano-particles and their interaction with nano-structured surfaces.

\section{Nomenclature}

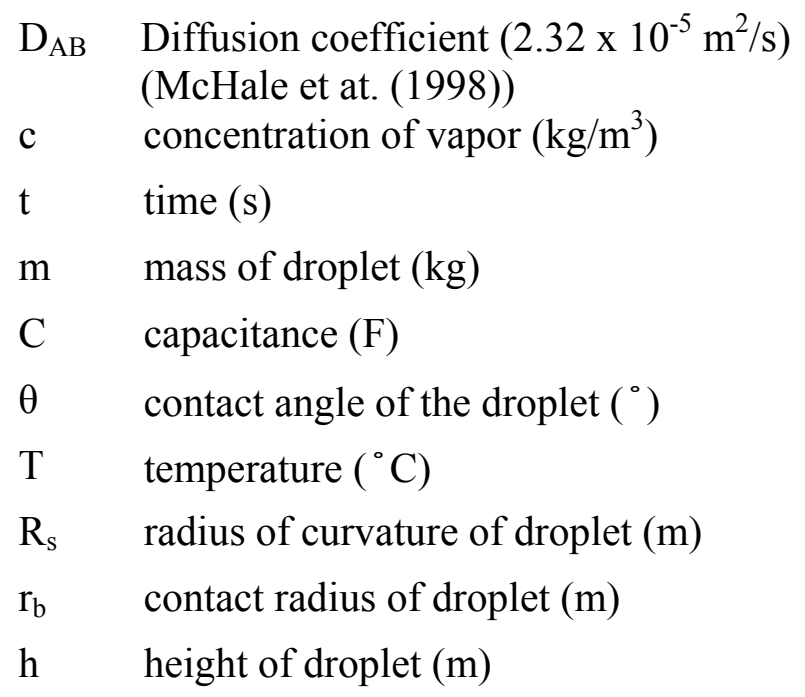

\section{Acknowledgement}

SK would like to acknowledge the fellowship program of the German Academic Exchange Service (DAAD), which facilitated his interaction on this topic of research with Prof. Peter Stephan at TU-Darmstadt, Germany.

The contribution of Mr. M. Siddhardh Chandra (M.Sc.) in design and fabrication of the experimental setup and its benchmarking is also acknowledged.

\section{References}

Bhardwaj, R., 2010. An Investigation of the Evaporation of a Droplet on a Solid Surface: Evaporation, SelfAssembly of Colloidal Deposits, and Interfacial Heat Transfer, PhD Thesis, Columbia University.

Burmeister, D., Badowsky, W., Barun, T., Wieprich, S., Boneberg, J., and Leiderer, P., 1999. Colloid monolayer lithography - a flexible approach for nanostructuring of surfaces, Appl. Surf. Sci. 144/145,461-466.

Bensimon, D., Simon, A. J., Croquette V., and Bensimon, A., 1995. Stretching DNA with a receding meniscus: experiments and models, Phy. Rev. Letts. 74, 4754-4757.
Choi, C. H., Kim, C. J., 2009. Droplet evaporation of pure water and protein solution on nanostructured superhydrophobic surfaces of varying heights, Langmuir 25(13), 7561-7567.

Deegan, R. D., Bakajin, O., Dupont, T. F., Huber, G., Nagel, S. R., and Witten, T. A., 1997. Capillary flow as the cause of ring stains from dried liquid drops, Nature $389,827-829$.

Erbil, H. Y., 2012. Evaporation of pure liquid sessile and spherical suspended drops: A review, Adv. Colloid Interfac. 170, 67-86.

Hu, H., Larson, R.G., 2002. Evaporation of a sessile droplet on a substrate, J. Phy. Chem. B 106, 1334-1344.

Lee, C. Y., Zhang, B. J., Park, J., Kim. K. J, 2012. Water droplet evaporation on $\mathrm{Cu}$ based hydrophobic surfaces with nano and micro-structures, Int. J. Heat Mass Transf. $55,2151-2159$.

McHale, G., Rowan, S. M., Newton, M. I., Banerjee, M. K., 1998. Evaporation and the wetting of a low-energy solid surface, J. Phy. Chem. B 102, 1964.

Maxwell, J. C., 1890. Diffusion, Collected scientific papers, Cambridge 2, 625.

Ondarcuhu, T., Joachim, C., 1998. Drawing a single nanofibre over hundreds of microns, Europhys Letts. 42, 215-220.

Picknett, R. G., Bexon, R., 1976. The evaporation of sessile or pendant drops in still air, J. Colloid Interface. Sci. 61 (2), 336-350.

Shmuylovich, L., Shen, A. Q., and Stone, H. A., 2002. Surface morphology of drying latex films: Multiple ring formation, Langmuir 18, 3441-3445.

Snow, C. J., 1949. Potential problems and capacitance for a conductor bounded by two intersecting spheres, J. Res. Nat. Bureau Standards (U. S. Deptt. of Commerce) 2032, 43, 377-407.

Wang, H., Wang, Z., Huang, L., Mitra, A., and Yan, Y., 2001. Surface patterned porous films by convectionassisted dynamic self-assembly of zeolite nanoparticles, Langmuir 17, 2572-2574. 